\title{
A two-stage approach for improved prediction of residue contact
} maps

\author{
Alessandro Vullo, Ian Walsh and Gianluca Pollastri*
}

\author{
Address: School of Computer Science and Informatics, University College Dublin, Belfield, Dublin 4, Ireland \\ Email: Alessandro Vullo - alessandro.vullo@ucd.ie; Ian Walsh - ian.walsh@ucd.ie; Gianluca Pollastri* - gianluca.pollastri@ucd.ie \\ * Corresponding author
}

Published: 30 March 2006

BMC Bioinformatics 2006, 7:180 doi:10.1186/1471-2105-7-180

This article is available from: http://www.biomedcentral.com/I47/-2/05/7//80

(C) 2006 Vullo et al; licensee BioMed Central Ltd.

This is an Open Access article distributed under the terms of the Creative Commons Attribution License (http://creativecommons.org/licenses/by/2.0), which permits unrestricted use, distribution, and reproduction in any medium, provided the original work is properly cited.
Received: 22 September 2005

Accepted: 30 March 2006

\begin{abstract}
Background: Protein topology representations such as residue contact maps are an important intermediate step towards $a b$ initio prediction of protein structure. Although improvements have occurred over the last years, the problem of accurately predicting residue contact maps from primary sequences is still largely unsolved. Among the reasons for this are the unbalanced nature of the problem (with far fewer examples of contacts than non-contacts), the formidable challenge of capturing long-range interactions in the maps, the intrinsic difficulty of mapping one-dimensional input sequences into two-dimensional output maps.

In order to alleviate these problems and achieve improved contact map predictions, in this paper we split the task into two stages: the prediction of a map's principal eigenvector (PE) from the primary sequence; the reconstruction of the contact map from the PE and primary sequence. Predicting the PE from the primary sequence consists in mapping a vector into a vector. This task is less complex than mapping vectors directly into two-dimensional matrices since the size of the problem is drastically reduced and so is the scale length of interactions that need to be learned.
\end{abstract}

Results: We develop architectures composed of ensembles of two-layered bidirectional recurrent neural networks to classify the components of the PE in 2, 3 and 4 classes from protein primary sequence, predicted secondary structure, and hydrophobicity interaction scales. Our predictor, tested on a non redundant set of $217 \mathrm{I}$ proteins, achieves classification performances of up to $72.6 \%, 16 \%$ above a base-line statistical predictor.

We design a system for the prediction of contact maps from the predicted PE. Our results show that predicting maps through the PE yields sizeable gains especially for long-range contacts which are particularly critical for accurate protein 3D reconstruction. The final predictor's accuracy on a non-redundant set of 327 targets is $35.4 \%$ and $19.8 \%$ for minimum contact separations of 12 and 24, respectively, when the top length/5 contacts are selected. On the II CASP6 Novel Fold targets we achieve similar accuracies (36.5\% and 19.7\%). This favourably compares with the best automated predictors at CASP6.

Conclusion: Our final system for contact map prediction achieves state-of-the-art performances, and may provide valuable constraints for improved $a b$ initio prediction of protein structures. A suite of predictors of structural features, including the PE, and PE-based contact maps, is available at http://distill.ucd.ie. 


\section{Background}

De novo prediction of protein three-dimensional (3D) structure from the primary sequence remains a fundamental and extraordinarily challenging problem [1]. Contact maps, or similar distance restraints have been proposed as intermediate steps between the primary sequence and the 3D structure (e.g. in [2-4]), for various reasons: unlike 3D coordinates, they are invariant to rotations and translations, hence less challenging to predict by machine learning systems [4]; quick, effective algorithms exist to derive 3D structures from them, for instance stochastic optimisation methods $[5,6]$, distance geometry $[7,8]$, or algorithms derived from the NMR literature and elsewhere [9-11]. Numerous methods have been developed for protein residue contact map prediction [2-4,12] and coarse (secondary structure element level) contact map prediction [13], and some improvements are slowly occurring (e.g. in [12], as shown by the CASP6 experiment [14]).

Still, accurate prediction of residue contact maps is far from being achieved and limitations of existing prediction methods have again emerged at CASP6 and from automatic evaluation of structure prediction servers such as EVA [15]. There are various reasons for this: the number of positive and negative examples (contacts vs. non contacts) is strongly unbalanced; the number of examples grows with the squared length of the protein making this a tough computational challenge; capturing long ranged interactions in the primary sequence is difficult, hence grasping an adequate global picture of the map is a formidable problem. For this reason simpler, alternative representations of protein topologies are particularly appealing, provided that they are informative and, especially, predictable (e.g. see [16]).

In this paper we focus on one such representation: the principal eigenvector (PE) of residue contact maps. The PE is a sequence of the same length as a protein's primary sequence. A vast machinery of tools for sequence processing is available (see e.g. [17] for a review). Moreover, recently [18] a branch-and-bound algorithm was described that is capable of reconstructing the contact map from the exact PE, at least for single domain proteins of up to 120 amino acids. This means that the PE contains most of the information encoded in the contact map. Predicting the PE is thus interesting: it leads to a drastic reduction in the size of the problem compared to twodimensional contact maps, i.e. considerable data compression, and also a reduction in the scale length of interactions that need to be learned; contact maps may be derived from the PE by modifying the reconstruction algorithm in [18] to deal with noise in the PE; alternatively the PE may be adopted as an additional input feature to systems for the direct prediction of contact maps (such as [4]); information contained in the PE may be used, in combination with other constraints, to guide the search for optimal 3D configurations; predicted PE may prove useful to identify domains, as in [19], and discussed in [20].

In this paper, we model the problem of inferring the PE as a classification task with multiple classes. We use machine learning methods to map amino acids into their corresponding component of the principal eigenvector. Similarly to [21], we adopt bidirectional recurrent neural networks (BRNNs) [22] with shortcut connections, accurate coding of input profiles obtained from multiple sequence alignments, secondary structure predictions, second stage filtering by recurrent neural networks, and finally large-scale ensembles of predictors. Our models classify correctly up to $72.6 \%$ residues, $16 \%$ above a baseline statistical predictor always assigning a residue to the most numerous PE class.

To prove that these levels can lead to improved contact maps, we incorporate the predicted PE into a state-of-theart system for contact map prediction $[4,13]$. Our tests show that the PE yields sizeable gains, and that these gains are especially significant for long-ranged contacts, which are known to be both harder to predict and critical for accurate $3 \mathrm{D}$ reconstruction.

\section{Results and discussion Principal eigenvector prediction}

We evaluate model performances using different prediction indices. If the task is the prediction of the eigenvector components $\bar{\lambda} \bar{x}_{i}$ (see the methods section for definitions) in $m$ classes, we measure: $Q_{m^{\prime}}$ or overall percentage of correctly predicted amino acids; the set $Q_{0}, \ldots, Q_{m-1}$, where each $Q_{j}$ is the percentage of correctly classified amino acids whose eigenvector component belongs to interval $I_{j+1}$; an analogous of the SOV measure [23] adapted for the case of $m$ classes. The intent in this case is to measure the quality of prediction over contiguous segments of amino acids belonging to the same class. Finally, we compare our methodology with a base-line predictor that assigns each amino acid to its most frequently occurring class (as for instance in [24-26]).

We train different ensembles of BRNNs. Differences depend on whether or not we use output filtering by second stage networks (Eq.6) and whether or not the input encoding includes predicted secondary structure from Porter [21] and the hydrophobicity interaction scale in [20] (Table I, column 2). Tables 1, 2 and 3 show respectively estimated performance indices for classification in two, three and four classes. The first three columns indicate whether secondary structure, hydrophobicity profile 
Table I: PE prediction: two-class problem. Accuracy estimates with $95 \%$ confidence intervals and SOV. A $*$ in the first three columns of a row indicates whether the results are obtained augmenting the network input with secondary structure predicted by Porter (P), hydrophobicity profile using the interactivity scale of [20] (H) and using second stage filtering network (F).

\begin{tabular}{|c|c|c|c|c|c|c|}
\hline$P$ & $\mathrm{H}$ & $\mathrm{F}$ & $Q_{2}$ & $Q_{0}$ & $Q_{1}$ & SOV \\
\hline - & - & - & $72.0 \pm .6$ & $73.1 \pm .6$ & $70.8 \pm .6$ & 44.4 \\
\hline- & - & $*$ & $72.1 \pm .6$ & $73.4 \pm .6$ & $70.7 \pm .6$ & 46.0 \\
\hline$*$ & - & - & $72.3 \pm .6$ & $73.1 \pm .6$ & $71.4 \pm .6$ & 47.6 \\
\hline$*$ & - & $*$ & $72.3 \pm .6$ & $72.8 \pm .6$ & $71.9 \pm .6$ & 49.6 \\
\hline$*$ & $*$ & - & $72.5 \pm .6$ & $74.0 \pm .6$ & $71.0 \pm .6$ & 47.2 \\
\hline \multirow[t]{2}{*}{$*$} & $*$ & $*$ & $72.6 \pm .6$ & $73.8 \pm .6$ & $71.2 \pm .6$ & 49.8 \\
\hline & baseline & & $56.8 \pm .5$ & $58.4 \pm .5$ & $55.1 \pm .5$ & - \\
\hline
\end{tabular}

and second stage filtering are employed in the network ensemble (see table legends for details).

In all multi-class prediction cases, the best network ensemble shows an increment of global predictive accuracy of $\approx 16 \%$ with respect to the base-line predictor. The SOV and the overall accuracy increase using filters and augmenting the number of input features with hydrophobicity scales (marginally) and secondary structure (significantly). Interestingly, predicted secondary structure is a valuable feature: in all cases, using true secondary structures results in only moderate improvements with respect to the performance obtained with secondary structure predicted by Porter [21] (data not shown).

In the 2-class problem, $Q_{2}$ exceeds $72 \%$ with the two classes almost equally well predicted. In this case, the network with full features finds a nearly optimal (Bayesian) decision threshold. This is not surprising because the threshold on the eigenvector component was chosen so as to divide the training set values in two equally distributed halves. The 3- and 4-class prediction problems are more difficult to solve, but the observed improvements over the baseline predictor are roughly the same as in the 2-class case. Strong improvements over the base-line predictor are observed especially for the intermediate classes $\left(Q_{1}\right.$ in Table 2, $Q_{1}$ and $Q_{2}$ in Table 3). Interestingly, these classes are more difficult to predict even if all classes are nearly equally distributed. This is possibly because boundary classes $\left(Q_{0}\right.$ and $\left.Q_{m-1}\right)$ correspond to well-defined situations, i.e. isolated residues or residues with high connectivity, for which clear signal exists in the data. A typical example of 4-class PE prediction is shown in figure 2.

\section{Contact map prediction from predicted PE}

As a final step, we test the possibility of directly using the information encoded in the PE to improve state-of-the-art residue contact map predictors. We choose the model based on DAG-RNNs described in [4] and [13]. This model was among the most successful contact map predictors at the CASP5 competition [27]. The architecture we adopt is identical to the one described in [13] and used at CASP5, except for the presence of shortcut connections and for the ensembling technique (see methods section). These differences allow a substantially (roughly 5-fold) faster training, and yield marginally improved results compared to [13] when the same input features and same training/test sets are adopted (not shown).

To ensure fairness, here we retrain DAG-RNNs from scratch using the same training and testing sets used to predict the PE. The sets are first processed to remove sequences longer than 200 amino acids (for computational reasons, as in [13]), leaving 1275 proteins in the training set and 327 proteins in the test set. Two amino acids are defined as being in contact if the distance between their $C_{\alpha}$ is below a contact threshold. We consider two different contact thresholds: 8 and $12 \AA$. For

Table 2: PE prediction: three-class problem. Accuracy estimates with $95 \%$ confidence intervals and SOV. A* in the first three columns of a row indicates whether the results are obtained augmenting the network input with secondary structure predicted by Porter $(P)$, hydrophobicity profile using the interactivity scale of $[20](H)$ and using second stage filtering network (F).

\begin{tabular}{|c|c|c|c|c|c|c|c|}
\hline$P$ & $\mathrm{H}$ & $F$ & $Q_{3}$ & $Q_{0}$ & $Q_{1}$ & $Q_{2}$ & sov \\
\hline- & - & - & $55.8 \pm .5$ & $63.7 \pm .5$ & $35.5 \pm .4$ & $67.6 \pm .6$ & 38.3 \\
\hline- & - & $*$ & $56.2 \pm .5$ & $61.6 \pm .6$ & $40.6 \pm .5$ & $65.8 \pm .6$ & 40.3 \\
\hline$*$ & - & - & $56.3 \pm .6$ & $65.8 \pm .6$ & $36.9 \pm .5$ & $65.5 \pm .6$ & 42.1 \\
\hline$*$ & - & $*$ & $56.6 \pm .6$ & $64.1 \pm .6$ & $41.1 \pm .4$ & $63.9 \pm .6$ & 43.6 \\
\hline$*$ & $*$ & - & $56.4 \pm .5$ & $65.2 \pm .6$ & $36.8 \pm .4$ & $66.4 \pm .6$ & 42.6 \\
\hline \multirow[t]{2}{*}{$*$} & $*$ & $*$ & $56.7 \pm .5$ & $63.3 \pm .6$ & $41.4 \pm .4$ & $64.6 \pm .6$ & 44.0 \\
\hline & baseline & & $39.8 \pm .4$ & $50.6 \pm .5$ & $8.5 \pm .2$ & $59.3 \pm .6$ & - \\
\hline
\end{tabular}


Table 3: PE prediction: four-class problem. Accuracy estimates with $95 \%$ confidence intervals and SOV. A $*$ in the first three columns of a row indicates whether the results are obtained augmenting the network input with secondary structure predicted by Porter $(P)$, hydrophobicity profile using the interactivity scale of $[20](H)$ and using second stage filtering network (F).

\begin{tabular}{ccccccccc}
\hline $\mathrm{P}$ & $\mathrm{H}$ & $\mathrm{F}$ & $Q_{4}$ & $Q_{0}$ & $Q_{1}$ & $Q_{2}$ & $Q_{3}$ & $\mathrm{SOV}$ \\
\hline- & - & - & $45.6 \pm .5$ & $59.9 \pm .6$ & $29.4 \pm .4$ & $26.8 \pm .4$ & $65.0 \pm .6$ & 33.2 \\
$*$ & - & $*$ & $46.0 \pm .5$ & $58.5 \pm .5$ & $30.6 \pm .4$ & $30.5 \pm .4$ & $63.3 \pm .6$ & 34.6 \\
$*$ & - & - & $46.2 \pm .5$ & $62.1 \pm .6$ & $30.5 \pm .4$ & $27.3 \pm .4$ & $63.1 \pm .6$ & 37.3 \\
$*$ & $*$ & $*$ & $46.5 \pm .5$ & $60.7 \pm .5$ & $32.3 \pm .4$ & $29.6 \pm .4$ & $61.8 \pm .5$ & 37.4 \\
$*$ & $*$ & - & $45.9 \pm .5$ & $61.5 \pm .5$ & $30.0 \pm .4$ & $27.3 \pm .3$ & $63.3 \pm .6$ & 37.1 \\
& baseline & & $46.5 \pm .5$ & $60.0 \pm .5$ & $32.2 \pm .4$ & $30.4 \pm .4$ & $61.9 \pm .6$ & 37.8 \\
& & & $30.7 \pm .4$ & $48.1 \pm .5$ & $5.4 \pm .2$ & $8.5 \pm .2$ & $59.0 \pm .6$ & - \\
\hline
\end{tabular}

comparison purposes, we encode each pair $(i, j)$ of amino acids in the input by four different features: a $20 \times 20$ matrix representing the probability distribution of pairs of amino acids observed in the two corresponding columns of the alignment (MA); MA plus the actual discretised 4class PE component for both residue $i$ and $j$ (MA_PE); MA plus the actual secondary structure ( 3 classes) and binary thresholded (at 25\%) relative solvent accessibility (MA_SS_ACC); and finally, the previous feature plus the actual 4-class PE components (MA_SS_ACC_PE). We train 8 predictors, with the same architecture, one for each input feature and contact threshold.

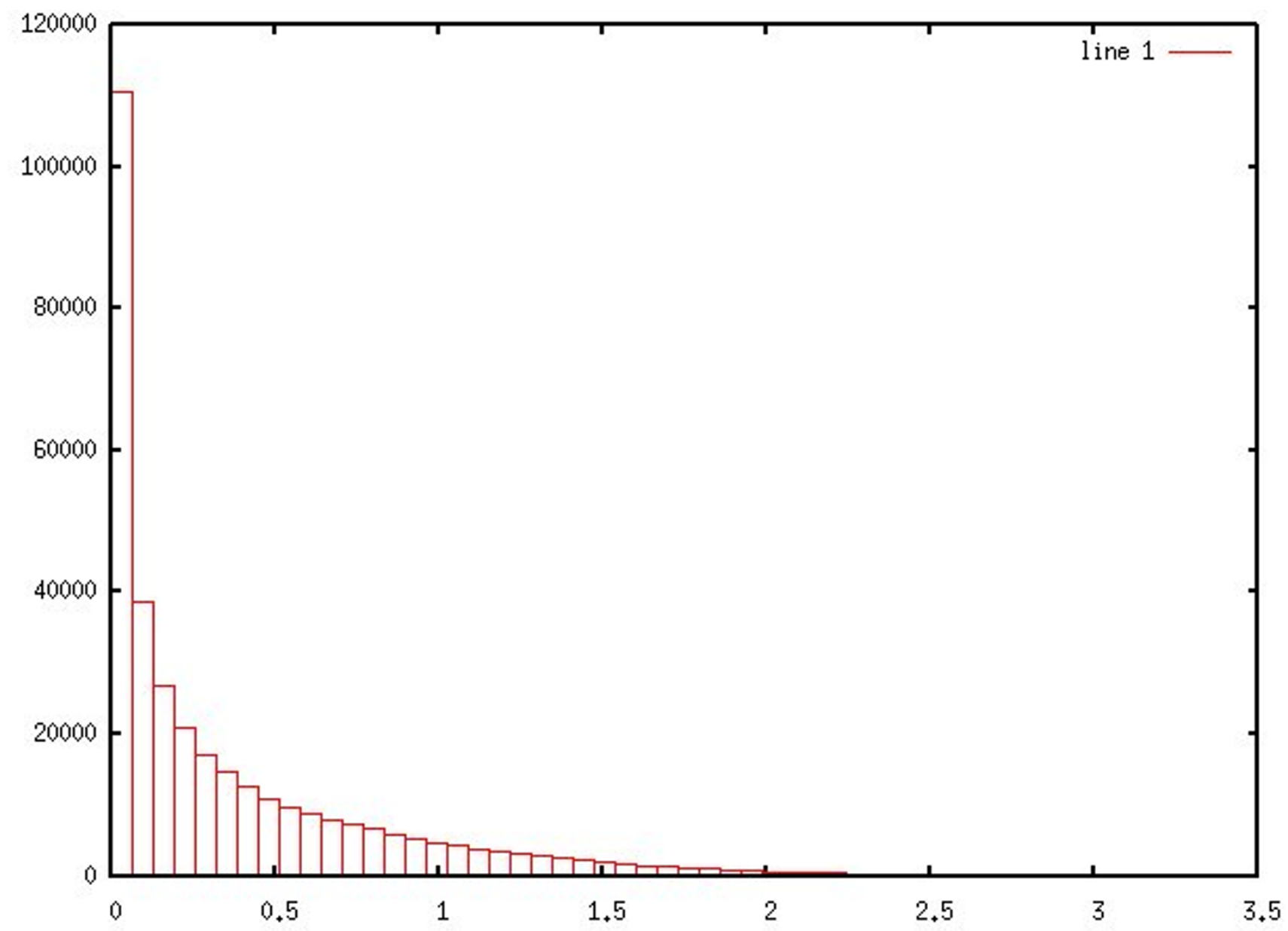

Figure I $\quad \bar{\lambda} \bar{x}$

Distribution of $\bar{\lambda} \bar{x}_{i}$ values in the training set. See text for details. 


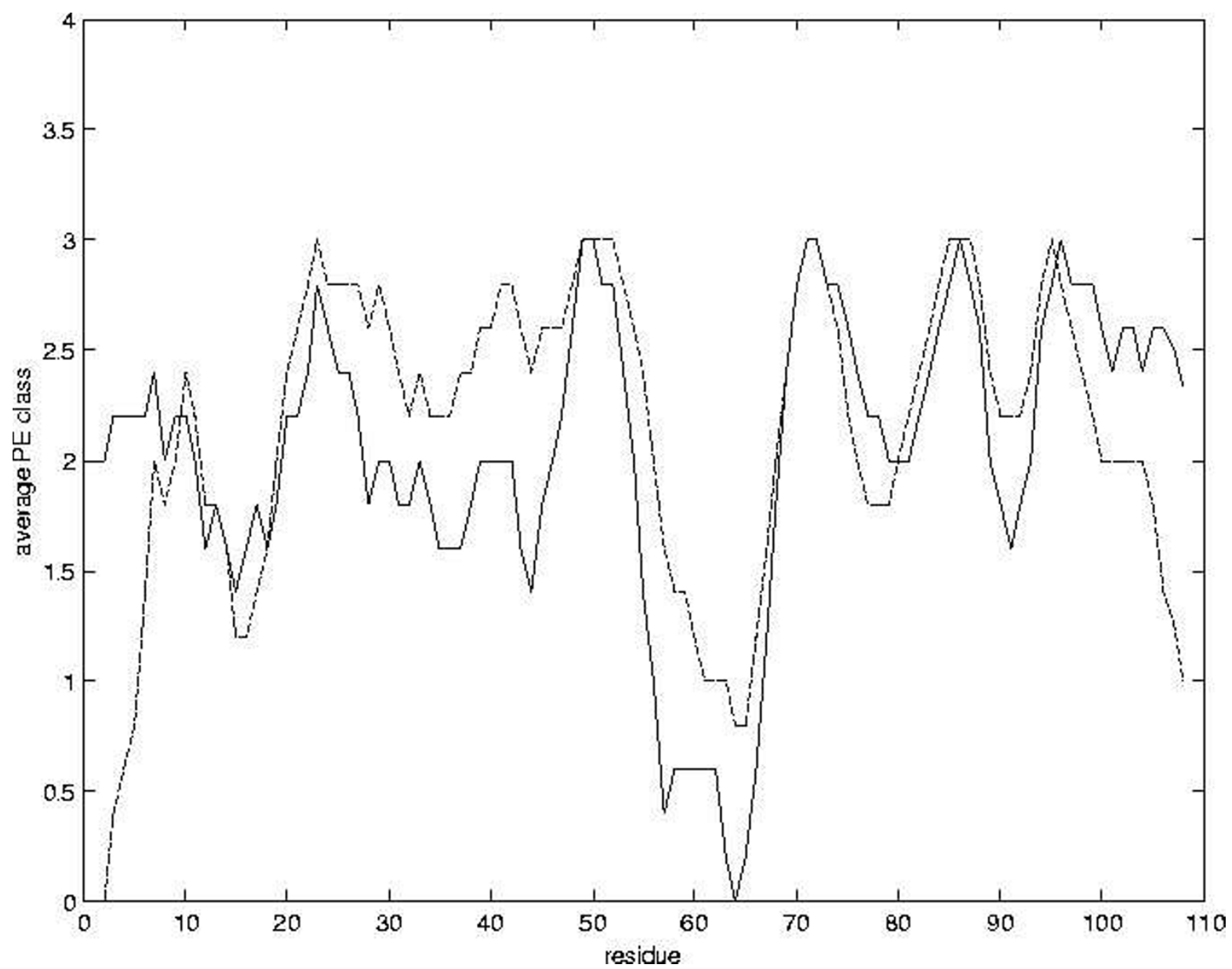

Figure 2

4-class principal eigenvector prediction for protein IA2P (108 amino acids). Solid line: exact eigenvector class. Dashed line: predicted eigenvector class. The class value is averaged over a moving window of 5 residues.

Differently from the training phase, testing takes place by encoding each pair $(i, j)$ on input with the predicted 4 -class PE component as given by the filtered ensemble of BRNNs using profiles, predicted secondary structure and hydrophobicity profiles (table 3, row P-H-F). Secondary structure and solvent accessibility information input into the DAG-RNN is also predicted during testing. These predictions are obtained from an architecture identical to the one adopted to predict the PE, and trained on the same training set. Hence the protocol we adopt leads to fully realistic results, since no protein in the the test set shows significant sequence similarity to any of the structures used to train the contact map predictor and all the underlying feature predictors.
Tables 4, 5, 6 and 7 show performance indices for all the 8 networks. Indices considered are: accuracy $P=T P /(T P+$ $F P)$, with $T P=$ true positives and $F P=$ false positives; coverage $R=T P /(T P+F N)$, with $F N=$ false negatives; $F_{1}$, defined as the harmonic mean of accuracy and coverage $\left(F_{1}=2 P R /(P+R)\right) ; P_{n c^{\prime}}$ the percentage of correctly predicted non-contacts. Performances are computed for three different sets of contacts, based on the separation of two residues in the linear sequence: $|i-j| \geq\{6,12,24\}$. In tables 4 and 6 we report $P, R$ and $F_{1}$ when the threshold between contacts and non-contacts is set to 0.5. In tables 5 and 7, for consistency with CASP assessment rules [14], we report $P$ and $R$ when only the top N/5 and N/2 contacts are considered, $N$ being the length of the protein. In this case contacts are ranked, and top contacts are selected, 
Table 4: Performance results for contact map prediction. Contact threshold: $8 \AA$ A. Accuracy, coverage and FI (as \%) for $8 \AA$ contact map predictor for distance separations greater than $5, I I$ and 23 amino acids.

\begin{tabular}{|c|c|c|c|c|c|c|c|c|c|c|c|c|}
\hline & \multicolumn{4}{|c|}{$|i-j| \geq 6$} & \multicolumn{4}{|c|}{$|i-j| \geq 12$} & \multicolumn{4}{|c|}{$|i-j| \geq 24$} \\
\hline & $P$ & $\mathrm{R}$ & $F_{1}$ & $P_{n c}$ & $P$ & $\mathrm{R}$ & $F_{1}$ & $P_{n c}$ & $P$ & $\mathrm{R}$ & $\mathrm{F}_{1}$ & $P_{n c}$ \\
\hline MA & 0 & 0 & 0 & 97.2 & 0 & 0 & 0 & 97.6 & 0 & 0 & 0 & 97.9 \\
\hline MA_PE & 39.4 & 12.2 & 18.6 & 97.5 & 36.2 & 8.4 & 13.5 & 97.7 & 27.8 & 2.0 & 3.7 & 97.9 \\
\hline MA_SS_ACC & 50.5 & 7.4 & 12.9 & 97.4 & 48.8 & 4.0 & 7.4 & 97.6 & 25.7 & .2 & .3 & 97.9 \\
\hline MA_SS_ACC_PE & 43.3 & 11.3 & 17.9 & 97.5 & 38.9 & 7.2 & 12.1 & 97.7 & 25.5 & 2.2 & 4.1 & 97.9 \\
\hline
\end{tabular}

based on their expected probability as estimated by the predictor.

As evident from the tables, the introduction of PE predictions increases the F1 measure in all cases. This is true for both 8 and 12 Å maps, and for all separation thresholds. An improvent is observed both in the MA_PE vs. MA case and in the MA_SS_ACC_PE vs. MA_SS_ACC case. In all cases the introduction of the predicted PE yields larger performance gains than secondary structure and solvent accessibility combined. Interestingly, the gains become more significant for longer range contacts. For instance for $|\mathrm{i}-\mathrm{j}| \geq 24 \mathrm{~F} 1$ grows from $0.3 \%$ to $4.1 \%$ at $8 \AA$ and from $5.3 \%$ to $16.8 \%$ at $12 \AA$ (MA_SS_ACC_PE vs. MA_SS_ACC). PE-based networks are more confident away from the main diagonal (a typical example is shown in figure 3), with a better balance between false positives and false negatives.

When we take into account only small numbers (N/5 and $N / 2$ ) of contacts considered most likely by the predictor, the gains become less marked, but remain significant, especially for longer range contacts: for $|i-j| \geq 24$ at $8 \AA$, when considering the top $N / 5$ contacts, $P$ grows from $14.1 \%$ to $19.6 \%$ in the MA vs. MA_PE case and from $17.8 \%$ to $19.8 \%$ in the in the MA_SS_ACC vs. MA_SS_ACC_PE case. Similar gains (from $43.3 \%$ to
$47.9 \%$ and from $43.7 \%$ to $49.9 \%$, respectively) are observed for the $12 \AA$ predictors.

Residue contact map predictors at CASP6 [14] were evaluated on a small set (11) of Novel Fold targets. The performances of the best system (group RR301) on the top $\mathrm{N} / 5$ contacts were $24 \%$ and $22 \%$ (accuracy) and $5.6 \%$ and $5 \%$ (coverage) for minimum residue separations of 12 and 24, respectively. Although the statistical relevance of a set of only 11 targets is limited, our predictor's accuracy on it compares favourably with the best CASP6 predictors, achieving $36.5 \%$ accuracy and $9.8 \%$ coverage for separation of at least 12 and $19.6 \%$ accuracy and $5.4 \%$ coverage for separation of at least 24 .

\section{Conclusion}

We developed sophisticated predictors of a novel sequential feature of protein structure: the principal eigenvector of residue contact maps. Our predictors classify correctly up to $72.6 \%$ of residues, and show large gains over simple base-line statistical predictors.

We showed that predicted principal eigenvectors can be effectively used as an additional input feature to a state-ofthe-art method for contact map prediction, yielding sizeable gains especially for long-range contacts which are particularly critical for accurate protein $3 \mathrm{D}$ reconstruction.

Table 5: Performance results for contact map prediction. Contact threshold: $8 \AA$ A. Accuracy and coverage (as \%) for $8 \AA$ contact map predictor for distance separations greater than $5, I I$ and 23 amino acids, when the top N/5 and top N/2 contacts are considered (where $N$ is the length of the protein).

\begin{tabular}{|c|c|c|c|c|c|c|c|c|c|c|c|c|}
\hline & \multicolumn{4}{|c|}{$|i-j| \geq 6$} & \multicolumn{4}{|c|}{$|i-j| \geq 12$} & \multicolumn{4}{|c|}{$|i-j| \geq 24$} \\
\hline & \multicolumn{2}{|c|}{$N / 5$} & \multicolumn{2}{|c|}{$N / 2$} & \multicolumn{2}{|c|}{$N / 5$} & \multicolumn{2}{|c|}{$N / 2$} & \multicolumn{2}{|c|}{$N / 5$} & \multicolumn{2}{|c|}{$N / 2$} \\
\hline & $P$ & $\mathrm{R}$ & $\mathrm{P}$ & $\mathrm{R}$ & $P$ & $\mathrm{R}$ & $P$ & $\mathrm{R}$ & $\mathrm{P}$ & $\mathrm{R}$ & $P$ & $\mathrm{R}$ \\
\hline MA & 30.8 & 3.9 & 26.2 & 8.5 & 23.9 & 3.8 & 19.2 & 7.8 & 14.1 & 3.3 & 11.1 & 6.6 \\
\hline MA_PE & 43.0 & 5.5 & 34.6 & 11.2 & 34.2 & 5.5 & 26.6 & 10.8 & 19.6 & 4.6 & 15.0 & 8.9 \\
\hline MA_SS_ACC & 44.4 & 5.7 & 36.0 & 11.6 & 34.2 & 5.5 & 26.6 & 10.8 & 17.8 & 4.2 & 14.7 & 8.7 \\
\hline MA_SS_ACC_PE & 46.4 & 5.9 & 36.6 & 11.8 & 35.4 & 5.7 & 27.0 & 11.0 & 19.8 & 4.6 & 15.7 & 9.3 \\
\hline
\end{tabular}


Table 6: Performance results for contact map prediction. Contact threshold: I 2 A. Accuracy, coverage and FI (as \%) for I Å contact map predictor for distance separations greater than $5, I I$ and 23 amino acids.

\begin{tabular}{|c|c|c|c|c|c|c|c|c|c|c|c|c|}
\hline & \multicolumn{4}{|c|}{$|i-j| \geq 6$} & \multicolumn{4}{|c|}{$|i-j| \geq 12$} & \multicolumn{4}{|c|}{$|i-j| \geq 24$} \\
\hline & $P$ & $\mathrm{R}$ & $\mathrm{F}_{\mathrm{I}}$ & $P_{n c}$ & $P$ & $\mathrm{R}$ & $F_{1}$ & $P_{n c}$ & $P$ & $\mathrm{R}$ & $\mathrm{F}_{1}$ & $P_{n c}$ \\
\hline MA & 60.4 & 10.6 & 18.1 & 87.2 & 55.8 & 0.1 & 0.1 & 87.8 & 38.9 & 0.03 & 0.06 & 88.8 \\
\hline MA_PE & 49.5 & 24.5 & 32.8 & 88.6 & 39.4 & 16.8 & 23.6 & 89.3 & 34.5 & 13.6 & 19.5 & 89.9 \\
\hline MA_SS_ACC & 61.6 & 19.6 & 29.7 & 88.2 & 48.9 & 7.5 & 13.1 & 88.5 & 40.2 & 2.8 & 5.3 & 89.0 \\
\hline MA_SS_ACC_PE & 54.2 & 23.5 & 32.8 & 88.6 & 42.2 & 14.6 & 21.7 & 89.2 & 36.7 & 10.9 & 16.8 & 89.7 \\
\hline
\end{tabular}

These results suggest a number of futher points to investigate:

- The algorithm in [18] may be directly tested in noisy contexts, and extended to increase its robustness. This may give rise to an alternative pipeline for the prediction of contact maps.

- The PE could be used directly to improve protein domain predictors $[19,20]$.

- PE-based maps may be adopted to guide the ab initio reconstruction of quick, draft $C_{\alpha}$ traces, for instance using a stochastic search algorithm similar to [5].

- Residue coordination number correlates well with the PE - as such, predicted coordination number [26] may yield similar gains to contact map prediction, while providing a more intuitive structural representation of a protein.

Ultimately, the third point is the most crucial test of the validity of our approach. Even if the 3D models produced were fairly coarse, they might be provide a valuable source of information, for instance to identify protein functions more accurately than it would be possible by sequence alone [28]. Although training a contact map prediction system is computationally expensive, once training is over, generating predictions is fast. Even on a small cluster of machines, this may allow multi-genomic scale structural prediction efforts in manageable times.

\section{Methods}

The contact map of a protein with $\mathrm{N}$ amino acids is a symmetric $N \times N$ matrix $C$, with elements $C_{i j}$ defined as:

$C_{i j}= \begin{cases}1 & \text { if amino acid } \mathrm{i} \text { and } \mathrm{j} \text { are in contact } \\ 0 & \text { otherwise }\end{cases}$

We define two amino acids as being in contact if the distance between their $C_{\alpha}$ is less than a given threshold. For the definition of the PE we adopt a fixed $8 \AA$ threshold, while in the contact map prediction stage we test $8 \AA$ and $12 \AA$ thresholds. Alternative definitions are possible, for instance based on different mutual $C_{\alpha}$ distances (normally in the 7-12 $\AA$ range), or on $C_{\beta} C_{\beta}$ atom distances (normally $6.5-8 \AA$ ), or on the minimal distance between two atoms belonging to the side-chain or backbone of the two residues (commonly $4.5 \AA$ ).

Let $\lambda(C)=\{\lambda: C x=\lambda x\}$ be the spectrum of $C, \mathcal{S}_{\lambda}=\{x$ : $C x=\lambda x\}$ the corresponding eigenspace and $\bar{\lambda}=\max \{\lambda \in$ $\lambda(C)\}$ the largest eigenvalue of $C$. The principal eigenvector of $C, \bar{x}$, is the eigenvector corresponding to $\bar{\lambda} . \bar{x}$ can also be expressed as the argument which maximises the Rayleigh quotient:

Table 7: Performance results for contact map prediction. Contact threshold: 12 A. Accuracy and coverage (as \%) for 12 A contact map predictor for distance separations greater than 5, I I and 23 amino acids, when the top N/5 and top N/2 contacts are considered (where $N$ is the length of the protein).

\begin{tabular}{|c|c|c|c|c|c|c|c|c|c|c|c|c|}
\hline & \multicolumn{4}{|c|}{$|i-j| \geq 6$} & \multicolumn{4}{|c|}{$|i-j| \geq 12$} & \multicolumn{4}{|c|}{$|i-j| \geq 24$} \\
\hline & \multicolumn{2}{|c|}{$N / 5$} & \multicolumn{2}{|c|}{$N / 2$} & \multicolumn{2}{|c|}{$N / 5$} & \multicolumn{2}{|c|}{$N / 2$} & \multicolumn{2}{|c|}{$N / 5$} & \multicolumn{2}{|c|}{$N / 2$} \\
\hline & $P$ & $\mathrm{R}$ & $P$ & $\mathrm{R}$ & $P$ & $\mathrm{R}$ & $P$ & $\mathrm{R}$ & $P$ & $\mathrm{R}$ & $P$ & $\mathrm{R}$ \\
\hline MA & 79.6 & 2.0 & 71.9 & 4.6 & 50.1 & 1.6 & 46.2 & 3.8 & 43.3 & 1.9 & 38.1 & 4.2 \\
\hline MA_PE & 87.5 & 2.2 & 81.6 & 5.2 & 59.8 & 1.9 & 54.1 & 4.4 & 47.9 & 2.1 & 42.4 & 4.7 \\
\hline MA_SS_ACC & 89.7 & 2.3 & 85.3 & 5.5 & 61.3 & 2.0 & 54.9 & 4.5 & 43.7 & 1.9 & 39.4 & 4.4 \\
\hline MA_SS_ACC_PE & 89.9 & 2.3 & 85.5 & 5.5 & 62.5 & 2.0 & 55.6 & 4.6 & 49.9 & 2.2 & 43.8 & 4.9 \\
\hline
\end{tabular}




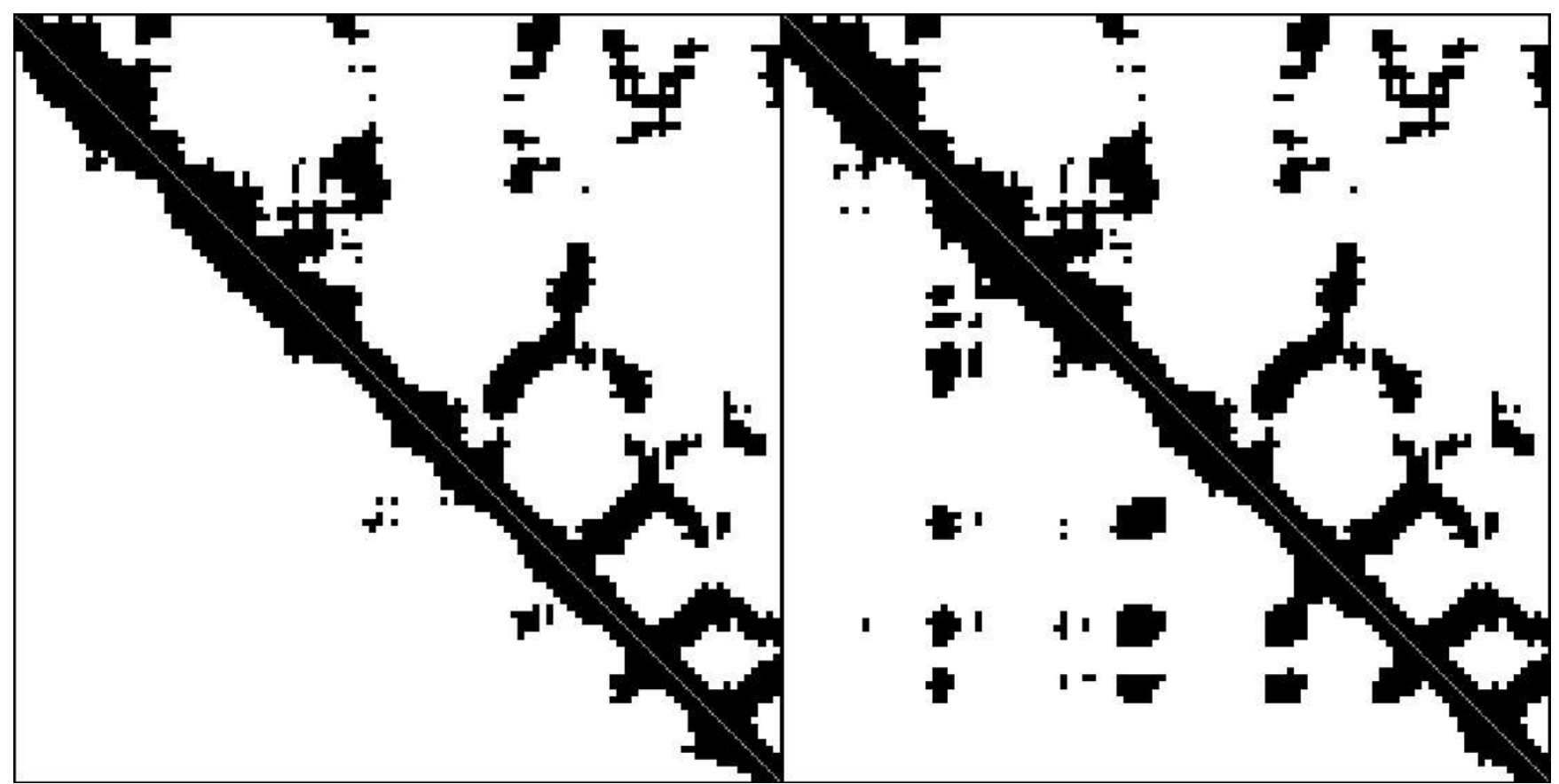

Figure 3

Examples of contact map predictions at I2 $\AA$ for protein IA2P (I 08 amino acids). Exact map in the top-right half, predicted map in the bottom-left half. Prediction by MA_SS_ACC on the left, MA_SS_ACC_PE on the right (see text for details).

$\forall x \in \mathcal{S}_{\lambda}: \frac{x^{T} C x}{x^{T} x} \leq \frac{\bar{x}^{T} C \bar{x}}{\bar{x}^{T} \bar{x}}$

Eigenvectors are usually normalised by requiring their norm to be 1, e.g. $\|x\|_{2}=1 \forall x \in \mathcal{S}_{\lambda}$. Since $C$ is an adjacency (real, symmetric) matrix, its eigenvalues are real. Since it is a normal matrix $\left(A^{H} A=A A^{H}\right)$, its eigenvectors are orthogonal. Other basic properties can also be proven: the principal eigenvalue is positive; non-zero components of $\bar{x}$ have all the same sign [29]. Without loss of generality, we can assume they are positive, as in [18].

Ideally, prediction of the PE should be formulated as a sequential regression task in which each amino acid is mapped into its corresponding component of the PE. Here we consider two variations to the original problem. First, we model it as a classification task with multiple classes. Second, we predict the magnified eigenvector, i.e.

$\bar{\lambda} \bar{x}$ instead of $\bar{x}$. Modelling regression problems as multi-class classifications is common practice, for instance in closely related tasks such as the prediction of protein solvent accessibility $[26,30,31]$. Predicting magnified eigenvector components has some advantages: by doing so we are simultaneously estimating the eigenvector components and the corresponding eigenvalue (as the norm of $\bar{x}$ is equal to 1 ); the main eigenvalue correlates well with the protein length (correlation of 0.62 on our training set), hence it is likely predictable. An estimate of the eigenvalue will in general be needed when attempting to predict contact maps from the $\mathrm{PE}$, either by using an algorithm similar to the one in [18], or more in general by attempting to satisfy the constraint:

$\left|\sum_{j} C_{i j} \bar{x}_{j}-\bar{\lambda} \bar{x}_{i}\right|=0$

Formally, the PE prediction task consists in learning a mapping $f(\cdot): \mathcal{I} \rightarrow O$ from the space $\mathcal{I}$ of labelled input sequences to the space $O$ of labelled output sequences. In practice, we want to predict a sequence of labels $O=\left(o_{1}, \ldots, o_{N}\right)$, for a given sequence of inputs $I=\left(i_{1}\right.$, $\left.\ldots, i_{N}\right)$, where each $i_{j} \in I$ is the input coding of the amino acid in position $j$. For PE prediction, we assume that there is a range $R$ including all magnified eigenvector components, i.e. $\forall j, \bar{\lambda} \bar{x}_{j} \in R$, and we divide the range $R$ into a series of $m$ disjoint intervals, i.e. $R=\bigcup_{k=1}^{m} R_{k}$. We can represent each output label $o_{j}$ as belonging to an alphabet 
of $m$ symbols, i.e. $o_{j} \in \Sigma=\{1, \ldots, m\}$, and $o_{j}$ corresponds to the class or interval $R_{k}$ in which the value of the $j$-th magnified eigenvector component falls: $o_{j}=k \Leftrightarrow \bar{\lambda} \bar{x}_{j} \in R_{k}$.

\section{Predictive architecture for the PE}

To learn the mapping between our inputs $\mathcal{I}$ and outputs $O$ we use a two-layered architecture composed of Bidirectional Recurrent Neural Networks (BRNN) [22] (also known as $1 \mathrm{D}-\mathrm{RNN}$, e.g. in [13]) of the same length as the amino acid sequence. Similarly to [21] we use BRNNs with shortcut connections. In these BRNNs, connections along the forward and backward hidden chains span more than 1-residue intervals, creating shorter paths between inputs and outputs. These networks take the form:

$$
\begin{aligned}
o_{j} & =\mathcal{N}^{(O)}\left(i_{j}, h_{j}^{(F)}, h_{j}^{(B)}\right) \\
h_{j}^{(F)} & =\mathcal{N}^{(F)}\left(i_{j}, h_{j-1}^{(F)}, \ldots, h_{j-S}^{(F)}\right) \\
h_{j}^{(B)} & =\mathcal{N}^{(B)}\left(i_{j}, h_{j+1}^{(B)}, \ldots, h_{j+S}^{(B)}\right) \\
j=1, \ldots, N &
\end{aligned}
$$

where $h_{j}^{(F)}$ and $h_{j}^{(B)}$ are forward and backward chains of hidden vectors with $h_{0}^{(F)}=h_{N+1}^{(B)}=0$. We parametrise the output update, forward update and backward update functions (respectively $\mathcal{N}(O), \mathcal{N}^{(F)}$ and $\mathcal{N}(B)$ using three two-layered feed-forward neural networks. In our tests the input associated with the $j$-th residue $i_{j}$ contains amino acid information, secondary structure information, and hydrophobicity interaction values described in [20]. Amino acid information is obtained from multiple sequence alignments of the protein sequence to its homologues to leverage evolutionary information. Amino acids are coded as letters out of an alphabet of 25. Beside the 20 standard amino acids, B (aspartic acid or asparagine), U (selenocysteine), X (unknown), Z (glutamic acid or glutamine) and (gap) are considered. The input presented to the networks is the frequency of each of the 24 non-gap symbols, plus the overall frequency of gaps in each column of the alignment. I.e., if $n_{j k}$ is the total number of occurrences of symbol $j$ in column $k$, and $g_{k}$ the number of gaps in the same column, the $j^{\text {th }}$ input to the networks in position $k$ is:

$$
\frac{n_{j k}}{\sum_{v=1}^{24} n_{v k}}
$$

for $j=1 \ldots 24$, while the $25^{\text {th }}$ input is:

$$
\frac{g_{k}}{g_{k}+\sum_{v=1}^{24} n_{v k}}
$$

This input coding scheme is richer than simple 20-letter schemes and has proven effective in [21]. The secondary structure part of the input is encoded using a three-letter scheme (helix, strand, coil). We adopt both true secondary structures, and secondary structures predicted by Porter [21]. When using predicted secondary structure, we carefully design our tests so that no sequence used for testing the PE prediction system is similar to sequences in Porter's training set. A single real-valued input is used to encode hydrophobicity interaction values. In [20] an optimised version of the scale is shown to be highly correlated to the PE. A further unit is used to encode the protein length (normalised by a factor 0.001).

Based on this encoding, a total of 30 units are used to represent each residue.

We adopt a second filtering BRNN, similarly to [21]. The network is trained to predict the PE given the first-layer PE predictions. The $i$-th input to this second network includes the first-layer predictions in position $i$ augmented by first stage predictions averaged over multiple contiguous windows. I.e., if $c_{j 1}, \ldots c_{j m}$ are the outputs in position $j$ of the first stage network corresponding to estimated probability of eigenvector component $j$ being in class $m$, the input to the second stage network in position $j$ is the array $I_{j}$ :

$$
\begin{array}{r}
\sum_{h=k_{-p}-w}^{I_{j}}=\left(c_{j 1}, \ldots, c_{j m},\right. \\
\sum_{h=k_{p}-w}^{k_{-p}+w} c_{h 1, \ldots,} \sum_{h=k_{-p}-w}^{k_{-p}+w} c_{h m,} \\
k_{p}+w \\
\left.\sum_{h=k_{p}-w}^{k_{p}+w} c_{h m}\right)
\end{array}
$$

where $k_{f}=j+f(2 w+1), 2 w+1$ is the size of the window over which first-stage predictions are averaged and $2 p+1$ is the number of windows considered. In the tests we use $w=7$ and $p=7$. This means that 15 contiguous, non-overlapping windows of 15 residues each are considered, i.e. first-stage outputs between position $j-112$ and $j+112$, for a total of 225 contiguous residues, are taken into account to 
generate the input to the filtering network in position $j$. This input contains a total of $16 \mathrm{~m}$ real numbers: $m$ representing the $m$-class output of the first stage in position $j$; $15 m$ representing the $m$-class outputs of the first-stage averaged over each of the 15 windows.

Five two-stage BRNN models are trained independently and ensemble averaged to build the final predictor. Differences among models are introduced by two factors: stochastic elements in the training-protocol, such as different initial weights of the networks and different shuffling of the examples; different architecture and number of free parameters of the models. Averaging the 5 models' outputs leads to classification performance improvements between $1 \%$ and $1.5 \%$ over single models. In [32] a slight improvement in secondary structure prediction accuracy was obtained by "brute ensembling" of several tens of different models trained independently. Here we adopt a less expensive technique: a copy of each of the 5 models is saved at regular intervals (100 epochs) during training. Stochastic elements in the training protocol (similar to that described in [33]) guarantee that differences during training are non-trivial. When an ensemble of 9 such copies for all the 5 models is used (45 models in total) we obtain a further slight improvement over the ensemble of 5 models.

\section{Predictive architecture for contact maps}

We build a system for the prediction of contact maps based on 2D-RNN, described in [4] and [13]. This is a family of adaptive models for mapping two-dimensional matrices of variable size into matrices of the same size. 2D-RNN-based models were among the most successful contact map predictors at the CASP5 competition [27].

As in the PE prediction case, we use 2D-RNNs with shortcut connections, i.e. where lateral memory connections span $N$-residue intervals, where $N>1$. If $o_{j, k}$ is the entry in the $j$-th row and $k$-th column of the output matrix (in our case, it will represent the estimated probability of residues $j$ and $k$ being in contact), and $i_{j, k}$ is the input in the same position, the input-output mapping is modelled as:

$$
\begin{aligned}
& o_{j, k}=\mathcal{N}^{(O)}\left(i_{j, k}, h_{j, k}^{(1)}, h_{j, k}^{(2)}, h_{j, k}^{(3)}, h_{j, k}^{(4)}\right) \\
& h_{j, k}^{(1)}=\mathcal{N}^{(1)}\left(i_{j, k}, h_{j-1, k^{\prime}, . .,}^{(1)} h_{j-S, k^{\prime}}^{(1)} h_{j, k-1}^{(1)}, . ., h_{j, k-S}^{(1)}\right) \\
& h_{j, k}^{(2)}=\mathcal{N}^{(2)}\left(i_{j, k}, h_{j+1, k^{\prime}}^{(2)}, h_{j+S, k^{\prime}}^{(2)}, h_{j, k-1}^{(2)}, \ldots, h_{j, k-S}^{(2)}\right) \\
& h_{j, k}^{(3)}=\mathcal{N}^{(3)}\left(i_{j, k}, h_{j+1, k^{\prime}}^{(3)}, h_{j+S, k}^{(3)}, h_{j, k+1}^{(3)}, . ., h_{j, k+S}^{(3)}\right) \\
& h_{j, k}^{(4)}=\mathcal{N}^{(4)}\left(i_{j, k}, h_{j-1, k^{\prime}, . .,}^{(4)} h_{j-S, k^{\prime}}^{(4)}, h_{j, k+1}^{(4)}, ., h_{j, k+S}^{(4)}\right) \\
& j, k=1, \ldots, N
\end{aligned}
$$

where $h_{j, k}^{(n)}$ for $n=1, \ldots, 4$ are planes of hidden vectors transmitting contextual information from each corner of the matrix to the opposite corner. We parametrise the output update, and the four lateral update functions (respectively $\mathcal{N}(O)$ and $\mathcal{N}(n)$ for $n=1, \ldots, 4)$ using five twolayered feed-forward neural networks, as in [13].

In our tests the input $i_{j, k}$ contains amino acid information, secondary structure and solvent accessibility information, and PE information for the amino acids in positions $j$ and $k$ in the sequence. Amino acid information is again obtained from multiple sequence alignments.

\section{Implementation}

\section{Data set generation and input data}

The data set used in the present simulations is extracted from the December 2003 25\% pdb_select list [34]. We use the DSSP program [35] (CMBI version) to assign relevant structural features (true secondary structure, used in preliminary tests, and $C_{\alpha}$ coordinates - the latter also directly available from the PDB files) and remove sequences for which DSSP does not produce an output due, for instance, to missing entries or format errors. After processing by DSSP, the set contains 2171 protein and 344,653 amino acids. Multiple sequence alignments for the 2171 proteins are extracted from the NR database as available on March 32004 containing over 1.4 million sequences. The database is first redundancy reduced at a $98 \%$ threshold, leading to a final 1.05 million sequences. The alignments are generated by three runs of PSI-BLAST [36] with parameters $b=3000, e=10^{-3}$ and $h=10^{-10}$.

\section{Experimental Protocol}

For our experiments we split the data into a training set containing 1736 sequences and a test set of 435 (1/5 of the total). The test set sequences are selected in an interleaved fashion (i.e. every fifth sequence is picked) from the whole set sorted alphabetically by PDB code - this is meant to avoid biases. 
For the prediction of contact maps the sets are further selected, by excluding proteins longer than 200 residues. This leaves 1275 proteins in the training set and 327 proteins in the test set.

To define the PE, two segments are considered in contact if the distance between their $C_{\alpha}$ is smaller than $8 \AA$. As in [18], the main diagonal of the contact map $(|i-j|<3)$ is removed before computing the principal eigenvectors. The distribution of the components of the magnified eigenvectors $\bar{\lambda} \bar{x}_{i}$ is shown in Figure 1 . We attempt three distinct classification schemes: in 2, 3 and 4 classes. The class thresholds are assigned so that the examples in the training set are equally split among them: 0.179195 for the 2-class case (classes of roughly 138, 000 examples each), 0.0688848 and 0.38165 for three classes $(\approx 92,000$ examples each) and 0.0358246, 0.179195 and 0.541445 for 4 classes $(\approx 69,000$ examples each). Both BRNNs are trained by minimising the cross-entropy error between the output and target probability distributions, using gradient descent with no momentum term or weight decay. The gradient is computed using the Back-propagation through structure (BPTS) algorithm (for which, see e.g. [37]). We use a hybrid between online and batch training, with 580 batch blocks (roughly 3 proteins each) per training set. Thus, the weights are updated 580 times per epoch. The training set is also shuffled at each epoch, so that the error does not decrease monotonically. When the error does not decrease for 50 consecutive epochs, the learning rate is divided by 2 . Training stops after 1000 epochs. First-layer and filtering BRNNs are trained simultaneously, but supervised independently.

The DAG-RNNs composing the contact map predictors are trained by minimising the cross-entropy error between the output and target probability distributions. This is obtained by a modified form of gradient descent where the update for a network weight is piecewise linear in three different ranges, to avoid initial plateau problems (for a more detailed description see [13]). The gradient is computed using the BPTS algorithm [37]. Similarly to the PE case, we use a hybrid between online and batch training, with 600 batch blocks (roughly 2 proteins each) per training set. Training runs for 120 epochs, with a fixed learning rate, and the training set is shuffled after each epoch. Three networks are saved, at epoch 110, 115 and 120 , and ensemble averaged to produce the final predictor.

Training the 8 predictors described in the paper took approximately a month on a cluster of $82.8 \mathrm{GHz}$ CPUs.

\section{Authors' contributions}

AV contributed the initial idea of predicting the principal eigenvector, and analysed the PE results. IW designed, trained and tested the predictor of contact maps. GP designed, trained and tested the PE predictive architecture, and suggested the two-stage approach for the prediction of contact maps. The manuscript was written by AV and GP, and read and approved by all authors.

\section{Acknowledgements}

We wish to thank Davide Baù for useful discussions, and Aoife McLysaght for her suggestions on how to improve the preliminary draft of this manuscript. This work is supported by Science Foundation Ireland grants 04/BR/ CS0353 and 05/RFP/CMS0029, grant RP/2005/219 from the Health

Research Board of Ireland, a UCD President's Award 2004, and an Embark Fellowship from the Irish Research Council for Science, Engineering and Technology to AV. We also wish to acknowledge the SFI/HEA Irish Centre for High-End Computing (ICHEC) for the provision of computational facilities and support.

\section{References}

I. Baker D, Sail A: Protein structure prediction and structural genomics. Science 200I, 294:93-96.

2. Fariselli P, Casadio R: A neural network based predictor of residue contacts in proteins. Protein Engineering I999, I 2(I): I5-2I.

3. Fariselli P, Olmea O, Valencia A, Casadio R: Prediction of contact maps with neural networks and correlated mutations. Protein Engineering 200 I, I 4( I I):835-439.

4. Pollastri G, Baldi P: Prediction of Contact Maps by Recurrent Neural Network Architectures and Hidden Context Propagation from All Four Cardinal Corners. Bioinformatics 2002, I 8(Suppl I):S62-S70.

5. Vendruscolo M, Kussell E, Domany E: Recovery of protein structure from contact maps. Folding and Design 1997, 2:295-306.

6. Debe D, Carlson M, Sadanobu J, Chan S, Goddard W: Protein fold determination from sparse distance restraints: the restrained generic protein direct Monte Carlo method. J Phys Chem 1999, I03:3001-3008.

7. Aszodi A, Gradwell M, Taylor W: Global fold determination from a small number of distance restraints. J Mol Biol 1995, 25 I:308-326.

8. Huang E, Samudrala R, Ponder J: Ab initio Fold Prediction of Small Helical Proteins Using Distance Geometry and Knowledge-Based Scoring Functions. J Mol Biol 1999, 290:267-28I.

9. Skolnick J, Kolinski A, Ortiz A: MONSSTER: a method for folding globular proteins with a small number of distance restraints. J Mol Biol 1997, 265:2 I7-24I.

10. Bowers P, Strauss C, Baker D: De novo protein structure determination using sparse NMR data. J Biomol NMR 2000, I 8:3||-3|8.

II. Li W, Zhang Y, Kihara D, Huang Y, Zheng D, Montelione G, Kolinski A, Skolnick J: TOUCHSTONEX: Protein structure prediction with sparse NMR data. Proteins: Structure, Function, and Genetics 2003, 53:290-306.

12. McCallum R: Striped sheets and protein contact prediction. Bioinformatics 2004, 20(Suppl I):224-23I.

13. Baldi P, Pollastri G: The Principled Design of Large- Scale Recursive Neural Network Architectures - DAG-RNNs and the Protein Structure Prediction Problem. Journal of Machine Learning Research 2003, 4(Sep):575-602.

14. CASP6 Home [http://predictioncenter.org/casp6/Casp6.html]

15. Eyrich V, Marti-Renom M, Przybylski D, Madhusudan M, Fiser A, Pazos F, Valencia A, Sali A, Rost B: EVA: continuous automatic evaluation od protein structure prediction servers. Bioinformatics 200I, I7:|242-|25|.

16. Kinjo AR, Nishikawa K: Recoverable one-dimensional encoding of three-dimensional protein structures. Bioinformatics 2005 , 2 I (10):2 167-2 I 70.

17. Baldi P, Brunak S: Bioinformatics: The Machine Learning Approach. Second200I. 
18. Porto M, Bastolla U, Roman H, Vendruscolo M: Reconstruction of protein structures from a vectorial representation. Phys Rev Lett 2004, 92:218101.

19. Holm L, Sander C: Parser for protein folding units. Proteins I994, 19:256-268

20. Bastolla U, Porto M, Roman H, Vendruscolo M: Principal eigenvector of contact matrices and hydrophobicity profiles in proteins. Proteins: Structure, Function, and Bioinformatics 2005, 58:22-30.

21. Pollastri G, McLysaght A: Porter: a new, accurate server for protein secondary structure prediction. Bioinformatics 2005, 2I(8): $1719-20$.

22. Baldi P, Brunak S, Frasconi P, Soda G, Pollastri G: Exploiting the past and the future in protein secondary structure prediction. Bioinformatics 1999, 1 5:937-946.

23. Zemla A, Venclovas C, Fidelis K, Rost B: A modified definition of Sov, a segment-based measure for protein secondary structure prediction assessment. Proteins 1999, 34:220-223.

24. Richardson C, Barlow D: The bottom line for prediction of residue solvent accessibility. Protein Engineering 1999, I 2: I05 I- 1054.

25. Fariselli P, Casadio R: Prediction of the number of residue contacts in proteins. Proceedings of the 2000 Conference on Intelligent Systems for Molecular Biology (ISMBOO), La Jolla, CA 2000:I46-I5I.

26. Pollastri G, Fariselli P, Casadio R, Baldi P: Prediction of Coordination Number and Relative Solvent Accessibility in Proteins. Proteins 2002, 47: 142-235.

27. Moult J, Fidelis K, Zemla A, Hubbard T: Critical assessment of methods of protein structure prediction (CASP)-round $\mathbf{V}$. Proteins 2003, 53(Suppl 6):334-339.

28. Bonneau R, Strauss C, Rohl C, Chivian D, Bradley P, Malmström L, Robertson T, Baker D, Sali A: De Novo Prediction of Threedimensional Structures for Major Protein Families. J Mol Biol 2002, 322:65-78.

29. Biggs N: Algebraic graph theory. Second 1994.

30. Rost B, Sander C: Conservation and prediction of solvent accessibility in protein families. Proteins 1994, 20(3):216-226.

31. Mucchielli-Giorgi M, Hazout S, Tuffery P: PredAcc: prediction of solvent accessibility. Bioinformatics 1999, I5(2): I76-I77.

32. Petersen T, Lundegaard C, Nielsen M, Bohr H, Bohr J, Brunak S, Gippert $\mathrm{G}$, Lund $\mathrm{O}$ : Prediction of protein secondary structure at 80\% accuracy. Proteins 2000, 4 I (I): I7-20.

33. Pollastri G, Przybylski D, Rost B, Baldi P: Improving the prediction of protein secondary structure in three and eight classes using recurrent neural networks and profiles. Proteins 2002, 47:228-235.

34. [http://bioinfo.tg.fh-giessen.de/pdbselect/].

35. Kabsch W, Sander C: Dictionary of protein secondary structure: pattern recognition of hydrogen-bonded and geometrical features. Biopolymers 1983, 22:2577-2637.

36. Altschul S, Madden T, Schaffer A: Gapped blast and psi-blast: a new generation of protein database search programs. Nucl Acids Res 1997, 25:3389-3402.

37. Frasconi $P$, Gori M, Sperduti A: A general framework for adaptive processing of data structures. IEEE Transactions on Neural Networks 1998, 9:768-786.

\section{Publish with Biomed Central and every} scientist can read your work free of charge

"BioMed Central will be the most significant development for disseminating the results of biomedical research in our lifetime. "

Sir Paul Nurse, Cancer Research UK

Your research papers will be:

- available free of charge to the entire biomedical community

- peer reviewed and published immediately upon acceptance

- cited in PubMed and archived on PubMed Central

- yours - you keep the copyright
BioMedcentral 